\title{
ALTERNATIVES TO THE GLOBAL WARMING POTENTIAL FOR COMPARING CLIMATE IMPACTS OF EMISSIONS OF GREENHOUSE GASES
}

\author{
KEITH P. SHINE ${ }^{1}$, JAN S. FUGLESTVEDT ${ }^{2}$, KINFE HAILEMARIAM $^{3}$ \\ and NICOLA STUBER ${ }^{4, *}$ \\ ${ }^{1}$ Department of Meteorology, The University of Reading, Reading, United Kingdom \\ E-mail:k.p.shine@reading.ac.uk \\ ${ }^{2}$ CICERO - Center for International Climate and Environmental Research, Oslo, Norway \\ ${ }^{3}$ National Meteorological Services Agency, Addis Ababa, Ethiopia \\ ${ }^{4}$ DLR - Institut für Physik der Atmosphäre, Oberpfaffenhofen, Germany
}

\begin{abstract}
The Global Warming Potential (GWP) is used within the Kyoto Protocol to the United Nations Framework Convention on Climate Change as a metric for weighting the climatic impact of emissions of different greenhouse gases. The GWP has been subjected to many criticisms because of its formulation, but nevertheless it has retained some favour because of the simplicity of its design and application, and its transparency compared to proposed alternatives. Here, two new metrics are proposed, which are based on a simple analytical climate model. The first metric is called the Global Temperature Change Potential and represents the temperature change at a given time due to a pulse emission of a gas $\left(\mathrm{GTP}_{\mathrm{P}}\right)$; the second is similar but represents the effect of a sustained emission change (hence $\mathrm{GTP}_{\mathrm{S}}$ ). Both $\mathrm{GTP}_{\mathrm{P}}$ and $\mathrm{GTP}_{\mathrm{S}}$ are presented as relative to the temperature change due to a similar emission change of a reference gas, here taken to be carbon dioxide. Both metrics are compared against an upwelling-diffusion energy balance model that resolves land and ocean and the hemispheres. The GTP $\mathrm{P}_{\mathrm{P}}$ does not perform well, compared to the energy balance model, except for

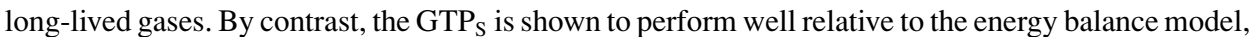
for gases with a wide variety of lifetimes. It is also shown that for time horizons in excess of about 100 years, the GTPS and GWP produce very similar results, indicating an alternative interpretation for the GWP. The GTPS retains the advantage of the GWP in terms of transparency, and the relatively small number of input parameters required for calculation. However, it has an enhanced relevance, as it is further down the cause-effect chain of the impacts of greenhouse gases emissions and has an unambiguous interpretation. It appears to be robust to key uncertainties and simplifications in its derivation and may be an attractive alternative to the GWP.
\end{abstract}

\section{Introduction}

The Intergovernmental Panel on Climate Change (IPCC) has, since its first scientific assessment in 1990, used the Global Warming Potential (GWP) as a method for comparing the potential climate impact of emissions of different greenhouse gases (IPCC, 1990; IPCC, 2001). The GWP is the time-integrated radiative forcing due to a pulse emission of a given gas, over some given time period (or horizon) relative to

*Current address: Department of Meteorology, The University of Reading, Reading, UK. 
a pulse emission of carbon dioxide (see Appendix A). The GWP has been adopted as an instrument in the Kyoto Protocol of the United Nations Framework Convention on Climate Change (UNFCCC). Emission targets are set in terms of equivalent emissions of carbon dioxide, where the carbon dioxide equivalence of emissions of other greenhouse gases (methane, nitrous oxide, the hydrofluorocarbons, the perfluorocarbons and sulphur hexafluoride) is determined using the GWP with a 100-year time horizon (henceforth GWP (100)).

Since its introduction, the GWP has been subject to scrutiny and criticism (e.g. Wigley, 1998; Fuglestvedt et al., 2000; O'Neill, 2000; Smith and Wigley, 2000a, b; Manne and Richels, 2001). One criticism relates to the fact that, despite its name, the global warming potential does not purport to represent the impact of gas emissions on temperature. The GWP uses the time-integrated radiative forcing and this does not give a unique indication of the effect of pulse emissions on temperature, because of large differences in the time constants of the various greenhouse gases. Although a strong greenhouse gas with a short lifetime could have the same GWP as a weaker greenhouse gas with a longer lifetime, identical (in mass terms) pulse emissions of the two gases could cause a different temperature change at a given time. Economists have also criticised the GWP concept for not being based on an analysis of damages caused by the emissions (e.g. Schmalensee, 1993; Kandlikar, 1995; Hammitt et al., 1996). Although within this paper we restrict the discussion to possible alternative physically based metrics, the new metrics could feed into the development of damage-based indices.

However, despite these criticisms and the suggestion of many alternatives, the GWP seems to have retained its attractiveness and widespread use, mainly because of the simplicity of its definition, the small number of required input parameters and the relative ease of calculation, compared to some of the alternatives. Additionally, the "transparency" and ease of application appear to be important aspects of acceptability amongst policymakers (Skodvin and Fuglestvedt, 1997; Fuglestvedt et al., 2003).

Preparations for the Fourth Assessment Report of the IPCC (due out in 2007) will begin soon as might negotiations over the second commitment period of the Kyoto Protocol (i.e. the period beyond 2012). This raises the question as to whether a metric can be designed that addresses some of the problems with the GWP, while at the same time maintaining its transparency and ease of use. This paper explores the use of two related metrics as a contribution to the growing debate (e.g. O'Neill, 2000; Smith and Wigley, 2000a, b; Godal, 2003; O'Neill, 2003; Smith, 2003) over whether, and how, the GWP could be superseded for use within international climate agreements and in other applications of climate change metrics.

The impact of climatically relevant emissions can be regarded, in a simplified manner, as the chain: emission changes $\rightarrow$ concentration changes $\rightarrow$ radiative forcing $\rightarrow$ climate impacts $\rightarrow$ societal and ecosystem impacts $\rightarrow$ economic "damage" 
(O'Neill, 2000; Smith and Wigley, 2000a; Fuglestvedt et al., 2003). It has been recognised that, in general, the relevance of the impacts becomes greater as we move down this chain, and hence it would be desirable to have a metric that compares an impact that is more relevant than the time-integrated radiative forcing. However, it has also been recognised that uncertainty generally becomes greater as we move down this chain.

This paper proposes alternatives to the GWP that move one step further down the chain from radiative forcing to represent the global-mean surface temperature change. It does so by using a simple model of the climate system, in the spirit of designing a transparent metric that may be more widely accepted. However, the framework we present is clearly suitable for extension beyond this by using, for example, output from a sophisticated climate model, and could incorporate other impacts, such as sea-level rise or economic damage, as an end point. Even if the proposed metrics are not acceptable as replacements for GWPs, the method does seem to have value as a pedagogic tool for testing alternative metrics and understanding the behaviour of various climate change agents and their effects on the climate system. We will call the new metrics the Global Temperature Change Potential for both a pulse emission $\left(\mathrm{GTP}_{\mathrm{P}}\right)$ and a sustained change in emissions $\left(\mathrm{GTP}_{\mathrm{S}}\right)$ of a gas.

It is somewhat surprising that such metrics have not been presented in the open literature before. The basic framework is well known (e.g. Hasselmann et al., 1997; Sausen and Schumann, 2000; Smith, 2003) and has been extensively used even as a way of assessing GWPs. An unpublished Technical Report by Meira Filho and Miguez (2000) proposed a wide range of possible metrics and included relationships similar to those presented in Section 2 - these authors did not, however, present numerical values or test the concept against more sophisticated models.

Within this paper we make an assumption that metrics are more likely to be widely accepted if they are available in a transparent and relatively simple analytical form; we then test these analytical forms against an energy balance model similar to that used by IPCC (2001). Of course, the output of such models, or even more sophisticated ones, could be used directly to generate values of indices such as the $\mathrm{GTP}_{\mathrm{P}}$ and $\mathrm{GTP}_{\mathrm{S}}$, without recourse to the analytical forms (see e.g. Smith, 2003); we refer to these as "exact" indices. Use of such models would allow inclusion of different scenarios of background concentrations of gases, nonlinear relationships between concentration and radiative forcing and even for more sophisticated treatment of the chemical processes affecting the pulse or change in emissions. The advantages of such "exact" indices would have to be balanced against likely barriers to their acceptance; the computer codes used to generate the indices would probably have to be agreed and maintained by a body such as IPCC, and be freely available in an "open source" form. 


\section{The Global Temperature Change Potentials}

The simplest representation of the global-mean surface temperature change, $\Delta T$, to a global-mean radiative forcing, $\Delta F$, is (e.g. Hartmann, 1996):

$$
C \frac{d \Delta T(t)}{d t}=\Delta F(t)-\frac{\Delta T(t)}{\lambda}
$$

where $t$ is time, $C$ is the heat capacity of the system and $\lambda$ is a climate sensitivity parameter, which indicates the change in equilibrium surface temperature per unit radiative forcing. In this paper it will be assumed that $\lambda$ is a constant that is independent of the particular mechanism causing the radiative forcing; however, the absolute value of $\lambda$ is poorly known (e.g. IPCC, 2001) and we will explore the dependence of the GTP metrics on this uncertainty. There is much current research in attempting to assess the extent to which $\lambda$ is truly independent of the radiative forcing mechanism (e.g. Hansen et al., 1997; Joshi et al., 2003) but it appears to be generally robust for the relatively well-mixed greenhouse gases being considered in the Kyoto Protocol. In addition, $\lambda$ may be dependent on the climate state (e.g. Raper et al., 2001) and this dependence is not included here. In any case, mechanism-dependent values of $\lambda$ could easily be incorporated within the framework of the GTP.

The general solution of (1) is

$$
\Delta T(t)=\frac{1}{C} \int_{0}^{t} \Delta F\left(t^{\prime}\right) \exp \left(\frac{t^{\prime}-t}{\lambda C}\right) d t^{\prime}
$$

where the exponential can be viewed as an impulse response (or Green) function to a $\delta$-function forcing at time $t^{\prime} . \lambda C$ is a time constant for the climate system and will be given the symbol $\tau$.

Such a simple model, as represented by Equation (1), can be used to obtain an approximate solution of the response to a forcing by regarding the heat capacity $C$ to represent the mixed layer of the ocean. The GTP $_{\mathrm{P}}$ and $\mathrm{GTP}_{\mathrm{S}}$ as presented here are, like the GWP, metrics that are relative to some reference gas. Hence, in assessing whether Equation (1) is fit for purpose, it is not the absolute accuracy that is an issue, but its ability to represent the temporal response of the global mean temperature relative to that of the reference gas. This important issue will be discussed in Sections 3 and 4.

\subsection{GTP FOR A PULSE EMISSION}

If $\Delta F(t)$ has a sufficiently simple form, Equation (2) can be integrated to yield an analytical form for the calculation of $\Delta T(t)$. We will consider first the Absolute Global Temperature Change Potential for a pulse emission, $\operatorname{AGTP}_{\mathrm{P}}$. The $\operatorname{AGTP}_{\mathrm{P}}$ indicates the temperature change at time $t$, due to a $1-\mathrm{kg}$ emission at $t=0$ and has units $\mathrm{K} \mathrm{kg}^{-1}$. 
For the majority of greenhouse gases, the radiative forcing following an emission at time $t=0$ will have the form $A \exp (-t / \alpha)$ where $\alpha$ is the time constant for removal of the gas from the atmosphere and $A$ is the radiative forcing for a 1-kg change in concentration of that gas. We will (following the IPCC procedure for calculating GWPs) assume that $A$ is independent of changes in that gas's concentration and that it is not dependent on the changes in concentration of other greenhouse gases, either because it is in sufficiently low concentrations or because a linearisation is made about present day concentrations. It will also be assumed that $\alpha$, the lifetime, is a constant, although, in reality, it depends on the concentration of the gas itself and other gases (such as the concentration of $\mathrm{OH}$ ). The issue of nonlinearity in $A$ and $\alpha$ is addressed elsewhere (e.g. IPCC, 1995; Wuebbles et al., 1995; Fuglestvedt et al., 2003; Smith and Wigley, 2003a, b) and can lead to systematic biases in the absolute value of metrics. The impact on relative metrics such as $\mathrm{GTP}_{\mathrm{P}}$ and $\mathrm{GTP}_{\mathrm{S}}$ is not so easy to predict and would need to be explored for a range of scenarios of future trace gas emissions.

In this case, using Equation (2) the $\operatorname{AGTP}_{\mathrm{P}}(t)$ for gas $\mathrm{x}$ is

$$
\operatorname{AGTP}_{\mathrm{P}}^{\mathrm{x}}(t)=\frac{A_{\mathrm{x}}}{C\left(\tau^{-1}-\alpha_{\mathrm{x}}^{-1}\right)}\left[\exp \left(-\frac{t}{\alpha_{\mathrm{x}}}\right)-\exp \left(-\frac{t}{\tau}\right)\right]
$$

when $\tau \neq \alpha_{\mathrm{x}}$. When $\tau=\alpha_{\mathrm{x}}$

$$
\operatorname{AGTP}_{\mathrm{P}}^{\mathrm{x}}(t)=\frac{A_{\mathrm{x}} t}{C} \exp \left(-\frac{t}{\alpha_{x}}\right) .
$$

For carbon dioxide, the concentration response to a pulse emission is more complex than the simple $\exp (-t / \alpha)$ form. In many applications this response, $R(t)$, derived from more complete carbon cycle models, has been approximated by

$$
R(t)=a_{\mathrm{o}}+\sum_{i} a_{i} \exp \left(-\frac{t}{\alpha_{i}}\right)
$$

where typically three or four terms are included in the summation. To provide compatibility with the GWP values presented in IPCC (2001), we use the fourterm representation derived from the Bern carbon cycle model (Joos et al., 1996) for the case of a constant future mixing ratio; the same response function, in a different mathematical form, was used in $\operatorname{IPCC}(1996,2001)$ - see the Appendix A for the coefficients and further details. Equation (4) is certainly suitable for the illustrative purposes of this paper; however, it does not incorporate known (but poorly quantified) nonlinearities in the carbon budget, such as terrestrial carbon cycle feedbacks. 
Incorporating the radiative forcing due to the pulse, $A^{\mathrm{C}} R(t)$, where $A^{\mathrm{C}}$ is the radiative forcing due to a $1-\mathrm{kg}$ change in carbon dioxide, into Equation (2) yields the $\mathrm{AGTP}_{\mathrm{P}}$ for carbon dioxide

$$
\begin{aligned}
\operatorname{AGTP}_{\mathrm{P}}^{\mathrm{C}}(t)= & \frac{A^{\mathrm{C}}}{C}\left\{\tau a_{\mathrm{o}}\left[1-\exp \left(-\frac{t}{\tau}\right)\right]\right. \\
& \left.+\sum_{i} \frac{a_{i}}{\left(\tau^{-1}-\alpha_{i}^{-1}\right)}\left[\exp \left(-\frac{t}{\alpha_{i}}\right)-\exp \left(-\frac{t}{\tau}\right)\right]\right\} .
\end{aligned}
$$

It has been the convention within IPCC to quote GWPs relative to $\mathrm{CO}_{2}$, although this choice is by no means an obvious one (e.g. Wuebbles et al., 1995). Following this convention, the relative pulse GTP for gas $\mathrm{x}$ is then

$$
\operatorname{GTP}_{\mathrm{P}}^{\mathrm{x}}(t)=\frac{\operatorname{AGTP}_{\mathrm{P}}^{\mathrm{x}}(t)}{\operatorname{AGTP}_{\mathrm{P}}^{\mathrm{C}}(t)} .
$$

Although the GTP follows the general philosophy of the GWP, a major distinction is that the final result is the ratio of the temperature changes at a particular time, $t$, rather than, as is the case for the GWP, the ratio of the integrated changes over the period leading up to $t$ (see Appendix A). Hence a pulse emission of $1 \mathrm{~kg}$ of gas $\mathrm{x}$ will give an identical temperature change in year $t$ as $\operatorname{GTP}_{\mathrm{P}}^{\mathrm{x}}(t)$ kilograms of carbon dioxide, at least to the extent that Equations (1), (3) and (5) are reasonable representations. As discussed earlier, the GWP does not guarantee any such equivalence.

\subsection{GTP FOR A SUSTAINED EMISSION CHANGE}

The GTP concept can be extended to consider the impact of sustained changes in emissions of a gas, a quantity that may arguably have greater policy relevance if a country were to make changes in a given industrial or agricultural process that had a long-term impact on emissions; at the very least the difference between the pulse and sustained forms is instructive. We denote this absolute GTP $_{S}$ as AGTP $_{S}$. The units of AGTPS are $\mathrm{K}\left(\mathrm{kg} \mathrm{year}^{-1}\right)^{-1}$ and it gives the change in temperature at time $t$, due to a constant $1 \mathrm{~kg} \mathrm{year}^{-1}$ increase in emissions between time $t=0$ and $t$.

For non- $\mathrm{CO}_{2}$ greenhouse gases, the concentration change $\Delta \chi$ resulting from a change in emissions $\Delta S$ can be represented by

$$
\frac{d \Delta \chi(t)}{d t}=\Delta S(t)-\frac{\Delta \chi(t)}{\alpha} .
$$

If $\Delta S$ is independent of time, it is straightforward to show that

$$
\Delta \chi(t)=\alpha \Delta S\left[1-\exp \left(-\frac{t}{\alpha}\right)\right],
$$


and then, by representing the forcing as $A \Delta \chi(t)$ and assuming that $\Delta S$ is unity (in $\mathrm{kg} \mathrm{year}^{-1}$ ), this can be incorporated into Equation (2) to yield the $\mathrm{AGTP}_{\mathrm{S}}$ for gas $\mathrm{x}$

$$
\begin{aligned}
\operatorname{AGTP}_{\mathrm{S}}^{\mathrm{x}}(t)= & \frac{\alpha_{\mathrm{x}} A_{\mathrm{x}}}{C}\left\{\tau\left[1-\exp \left(-\frac{t}{\tau}\right)\right]\right. \\
& \left.-\frac{1}{\left(\tau^{-1}-\alpha_{\mathrm{x}}^{-1}\right)}\left[\exp \left(-\frac{t}{\alpha_{\mathrm{x}}}\right)-\exp \left(-\frac{t}{\tau}\right)\right]\right\}
\end{aligned}
$$

when $\tau \neq \alpha_{\mathrm{x}}$. When $\tau=\alpha_{\mathrm{x}}$

$$
\operatorname{AGTP}_{\mathrm{S}}^{\mathrm{x}}(t)=\frac{\alpha_{\mathrm{x}} A_{\mathrm{x}}}{C}\left\{\alpha_{\mathrm{x}}\left[1-\exp \left(-\frac{t}{\alpha_{\mathrm{x}}}\right)\right]-t \exp \left(-\frac{t}{\alpha_{\mathrm{x}}}\right)\right\} .
$$

The AGTP $\mathrm{A}_{\mathrm{S}}$ for $\mathrm{CO}_{2}$ is more complicated because of the nature of its response function (Equation (4)); a simplified representation can be given by the analogue to Equation (2) for the case of modelling the time-dependent response to changes in $\mathrm{CO}_{2}$ emissions. Using Equation (4), the response to a series of pulse emissions is given by

$$
\Delta \chi^{\mathrm{C}}(t)=\int_{0}^{t} \Delta S^{\mathrm{C}}\left(t^{\prime}\right)\left[a_{\mathrm{o}}+\sum_{i} a_{i} \exp \left(\frac{t^{\prime}-t}{\alpha_{i}}\right)\right] d t^{\prime}
$$

where the superscript $\mathrm{C}$ refers to carbon dioxide.

This can be incorporated into Equation (2), again assuming $\Delta S^{\mathrm{C}}$ is unity, to yield the $\mathrm{AGTP}_{\mathrm{S}}$ for carbon dioxide

$$
\begin{aligned}
\operatorname{AGTP}_{\mathrm{S}}^{\mathrm{C}}(t)= & \frac{A_{\mathrm{C}}}{C}\left\{a_{\mathrm{o}} t \tau-a_{\mathrm{o}} \tau^{2}\left[1-\exp \left(-\frac{t}{\tau}\right)\right]\right. \\
& +\sum_{i} \alpha_{i} a_{i}\left[\tau\left(1-\exp \left(-\frac{t}{\tau}\right)\right)\right. \\
& \left.\left.-\frac{1}{\tau^{-1}-\alpha_{i}^{-1}}\left(\exp \left(-\frac{t}{\alpha_{i}}\right)-\exp \left(-\frac{t}{\tau}\right)\right)\right]\right\} .
\end{aligned}
$$

The relative $\operatorname{GTP}_{\mathrm{S}}^{\mathrm{x}}$ is then

$$
\operatorname{GTP}_{\mathrm{S}}^{\mathrm{x}}(t)=\frac{\operatorname{AGTP}_{\mathrm{S}}^{\mathrm{x}}(t)}{\operatorname{AGTP}_{\mathrm{S}}^{\mathrm{C}}(t)} .
$$

Although somewhat more complicated than the equivalent GWP expressions, Equations (3), (5), (9) and (11) are nevertheless transparent in their derivation, require relatively few parameters to calculate (note that while the heat capacity $C$ cancels in Equations (6) and (12), it is still required for the calculation of $\tau$ ) and are suitable for use by policymakers and other parties with little or no further scientific input. The AGTP values for carbon dioxide could, of course, be derived using a more sophisticated carbon cycle model and provided as values at given time 
horizons, in place of Equations (5) and (11); these values could then be used in the denominator for GTP calculations.

Some care must be taken with the units when calculating the absolute values as, for example, radiative forcings are often quoted per ppbv rather than per unit mass, and because of the choice of years as the basic time unit. Appendix A elaborates on the necessary constants required to derive consistent values.

\section{Assessment of the Performance of $\operatorname{GTP}_{P}$ Relative to an Energy Balance Model}

Clearly the model embodied in Equation (1) is a gross simplification of the climate system. One of the simplifications is that the thermal inertia of the climate system is represented by that of the ocean mixed layer so that the climate system has a single time constant. Transfer of heat into the deep ocean, by diffusive and convective processes, slows the climate system response but also adds to the memory of the system's response to a pulse, while the response of the land surface adds a fast component. The ability of Equation (1) to calculate values of $\mathrm{GTP}_{\mathrm{P}}$ and $\mathrm{GTP}_{\mathrm{S}}$ is explored using an energy balance climate model (EBM), similar to that used within IPCC assessments (see IPCC, 2001). The EBM is used as a replacement for Equation (1), but using the same representations of the trace gas forcing and lifetimes as in the calculation of the analytical GTP. The EBM incorporates an upwelling-diffusion model for the ocean and it resolves land and oceans separately in each hemisphere. The parameters used here are described in Appendix B. Of course, caution must be expressed as to the ability of the EBM to represent the true system response, but Raper et al. (2001) present evidence that these models are able to simulate the surface temperature response from coupled atmosphere-ocean GCMs.

For the analytical calculations of GTP $_{P}$ and GTP $_{S}$ using the expressions derived in Section 2, it is assumed that the heat capacity $C$ is that appropriate to a global ocean mixed layer of $100-\mathrm{m}$ depth (i.e. $4.2 \times 10^{8} \mathrm{~J} \mathrm{~K}^{-1} \mathrm{~m}^{-2}$ ) and $\lambda$ is $0.8 \mathrm{~K}$ $\left(\mathrm{W} \mathrm{m}^{-2}\right)^{-1}$, appropriate to an equilibrium surface warming of $3 \mathrm{~K}$ for a doubling of $\mathrm{CO}_{2}$. This parameter choice yields a value of $\tau$ of 10.7 years. There is some scope for tuning the value of $C$ to best agree with the EBM, but the best-fit value of $C$ was found to vary with the chosen time horizon and, to some extent, with the gas under consideration.

Table I shows the AGWP and $\mathrm{AGTP}_{\mathrm{P}}$ for carbon dioxide at the three time horizons (20, 100 and 500 years) routinely reported by IPCC; the $\mathrm{AGTP}_{\mathrm{P}}$ is calculated using both the analytical expressions and the EBM. It also shows the values of the GWP and GTP $_{P}$ for five gases with a wide range of properties: HFC152a is chosen as a very short-lived gas in quite widespread use; methane is, after carbon dioxide, the second most important greenhouse gas (in terms of total radiative forcing since pre-industrial times) included within the Kyoto Protocol; HFC134a is the dominant hydrofluorocarbon in terms of its contribution to radiative forcing; $\mathrm{N}_{2} \mathrm{O}$ is a relatively long-lived gas, again important in terms of its contribution to 


\section{TABLE I}

AGWP (in $10^{-14} \mathrm{~W} \mathrm{~m}^{-2} \mathrm{~kg}^{-1}$ year) and $\operatorname{AGTP}_{\mathrm{P}}\left(\right.$ in $10^{-16} \mathrm{~K} \mathrm{~kg}^{-1}$ ) from both the analytical equations and the energy balance model (EBM) for carbon dioxide, and the GWP and $\mathrm{GTP}_{\mathrm{P}}$ for five other greenhouse gases at time horizons of 20, 100 and $500 \mathrm{yr}$

\begin{tabular}{|c|c|c|c|c|c|c|c|c|c|}
\hline & \multicolumn{3}{|c|}{ GWP } & \multicolumn{3}{|c|}{ Analytical GTP ${ }_{P}$} & \multicolumn{3}{|c|}{$\mathrm{EBM} \mathrm{GTP}_{\mathrm{P}}$} \\
\hline & 20 & 100 & 500 & 20 & 100 & 500 & 20 & 100 & 500 \\
\hline Absolute $\mathrm{CO}_{2}$ & 2.66 & 9.05 & 29.1 & 8.34 & 5.46 & 3.47 & 5.38 & 4.55 & 3.38 \\
\hline HFC152a & 400 & 120 & 37 & 170 & 0.15 & 0 & 135 & 22 & 4.0 \\
\hline $\mathrm{CH}_{4}$ & 62 & 22 & 7 & 52 & 0.35 & 0 & 46 & 5 & 0.8 \\
\hline HFC134a & 3290 & 1260 & 390 & 2840 & 34 & 0 & 2550 & 300 & 44 \\
\hline $\mathrm{N}_{2} \mathrm{O}$ & 270 & 290 & 150 & 290 & 270 & 13 & 290 & 270 & 35 \\
\hline $\mathrm{CF}_{4}$ & 3850 & 5650 & 8730 & 4150 & 7490 & 11700 & 4320 & 7090 & 11200 \\
\hline
\end{tabular}

Note. The values for methane include the indirect forcing. The analytical GTP values are calculated with a climate sensitivity of $0.8 \mathrm{~K}\left(\mathrm{~W} \mathrm{~m}^{-2}\right)^{-1}$ and a mixed layer with a depth of $100 \mathrm{~m}$. The EBM GTP values are derived using the same climate sensitivity, with other parameters given in Appendix B.

radiative forcing; and $\mathrm{CF}_{4}$ is a representative of the very long-lived greenhouse gases. For all these gases, lifetimes and the radiative forcing per ppbv are taken from IPCC (2001), to ensure that the GWPs calculated here essentially agree with values given in that report, although there are grounds for revising some of these values (see e.g. Sihra et al., 2001); the adopted values, and an explanation for a slight deviation from GWPs in IPCC (2001), are given in Appendix A. The AGWP and $\operatorname{AGTP}_{\mathrm{P}}$ for methane have been multiplied by 1.3 to account for the indirect forcing from tropospheric ozone and stratospheric water vapour changes, again following IPCC (2001). Figure 1a shows the radiative forcing due to the pulse emission for a selection of the gases considered here, and Figure 1b shows the respective temperature response to these pulse emissions using both the analytical and EBM-generated values of the AGTP $_{\mathrm{P}}$. Note that to reduce the number of lines on these plots, HFC134a and $\mathrm{N}_{2} \mathrm{O}$ are not included. However, given that HFC134a has a lifetime similar to that of methane (Table AI in Appendix A), the HFC134a curve is almost identical to the methane curve ignoring the multiplication by 50 (which is the ratio of the specific radiative forcings per unit mass of HFC134a to methane, including the methane indirect effects).

Turning now to the relative GTP $_{P}$, Table I presents the values while Figure $2 \mathrm{a}$ shows the percentage error in the analytical GTP $\mathrm{P}_{\mathrm{P}}$ relative to the $\mathrm{GTP}_{\mathrm{P}}$ calculated using the EBM.

Figure 1b shows how the simplifications in the analytical AGTP do not allow it to capture important features of the response that are found with the EBM. During the first few years after the pulse emission, the EBM temperature change is higher because of its rapidly responding land component. At longer times, except for the very short-lived HFC152a and $\mathrm{CO}_{2}$, Equation (1) both overestimates the peak response and underestimates the recovery from the pulse. Both these features are 


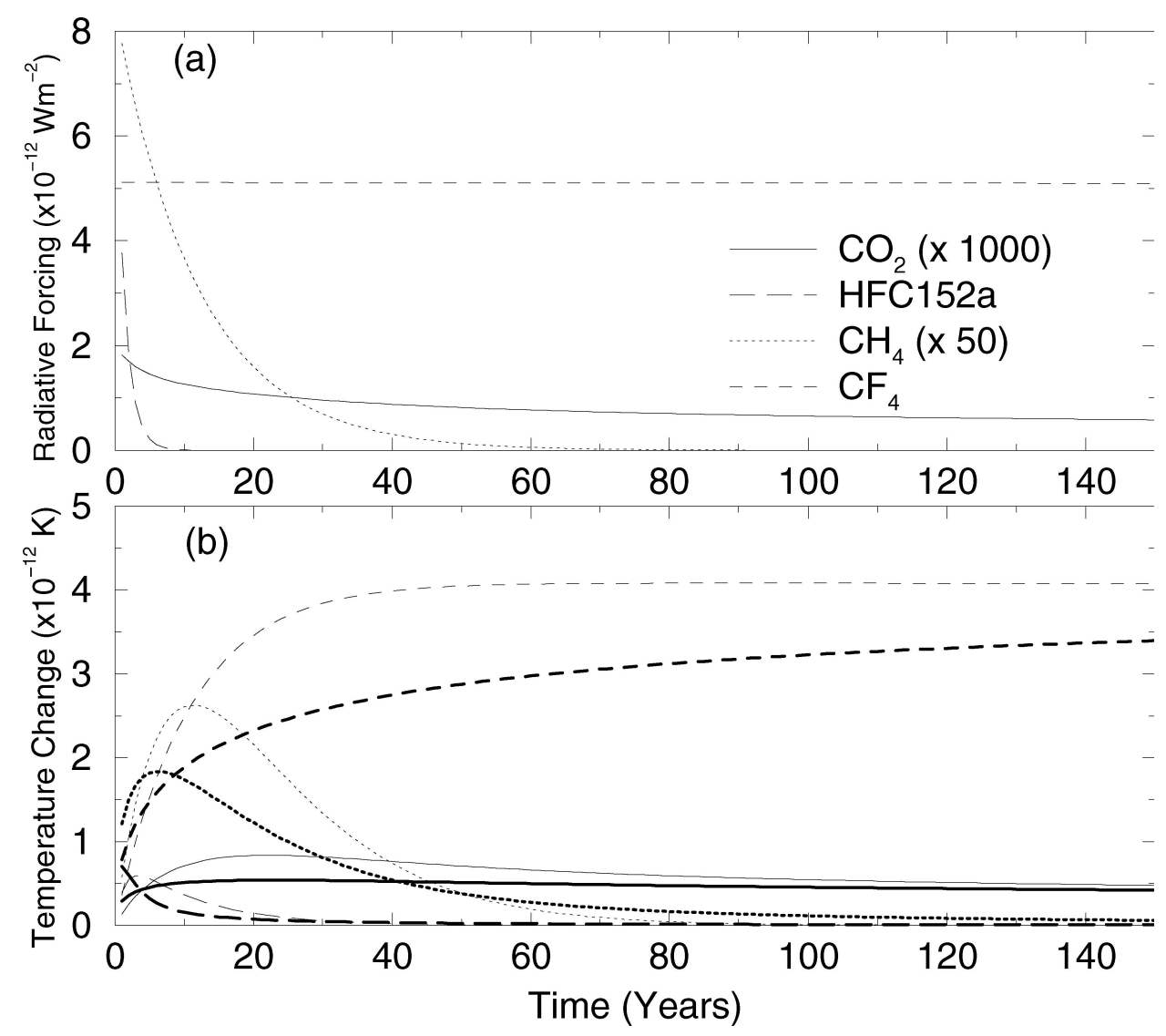

Figure 1. (a) Radiative forcing due to a 1-kg pulse emission of greenhouse gases with a range of lifetimes (see Table AI). The AGWP is the integral under these curves to a given time horizon. (b) Temperature response using the analytical $\operatorname{AGTP}_{\mathrm{P}}$ (thin lines) and the Energy Balance Model (thick lines) to the radiative forcing shown in (a).

because the EBM has a larger thermal inertia because of its deeper ocean, which mutes the initial response but maintains a longer memory of the pulse.

For the shorter time horizon of 20 years, Table I shows that the analytical and EBM values agree reasonably well, although for HFC152a this is just a coincidence, as Figure 2a shows it is close to the point in the change in sign of its error. At 100 years, it can be seen that for the shorter-lived greenhouse gases there is a substantial difference between the two GTP $_{P}$ values, by an order of magnitude or more - this is because the deep ocean prolongs the relative temperature response for the shortlived gases in the EBM. Further tests revealed that for the analytical GTP $\mathrm{P}_{\mathrm{P}}$ to agree within $10 \%$ of the EBM GTP at 100 years, gases need to have a lifetime of about 80 years or more.

Table I and Figure 1 show the result that has been pointed out elsewhere (e.g. Smith and Wigley, 2000a; Fuglestvedt, et al., 2003) that the GWP does not describe 

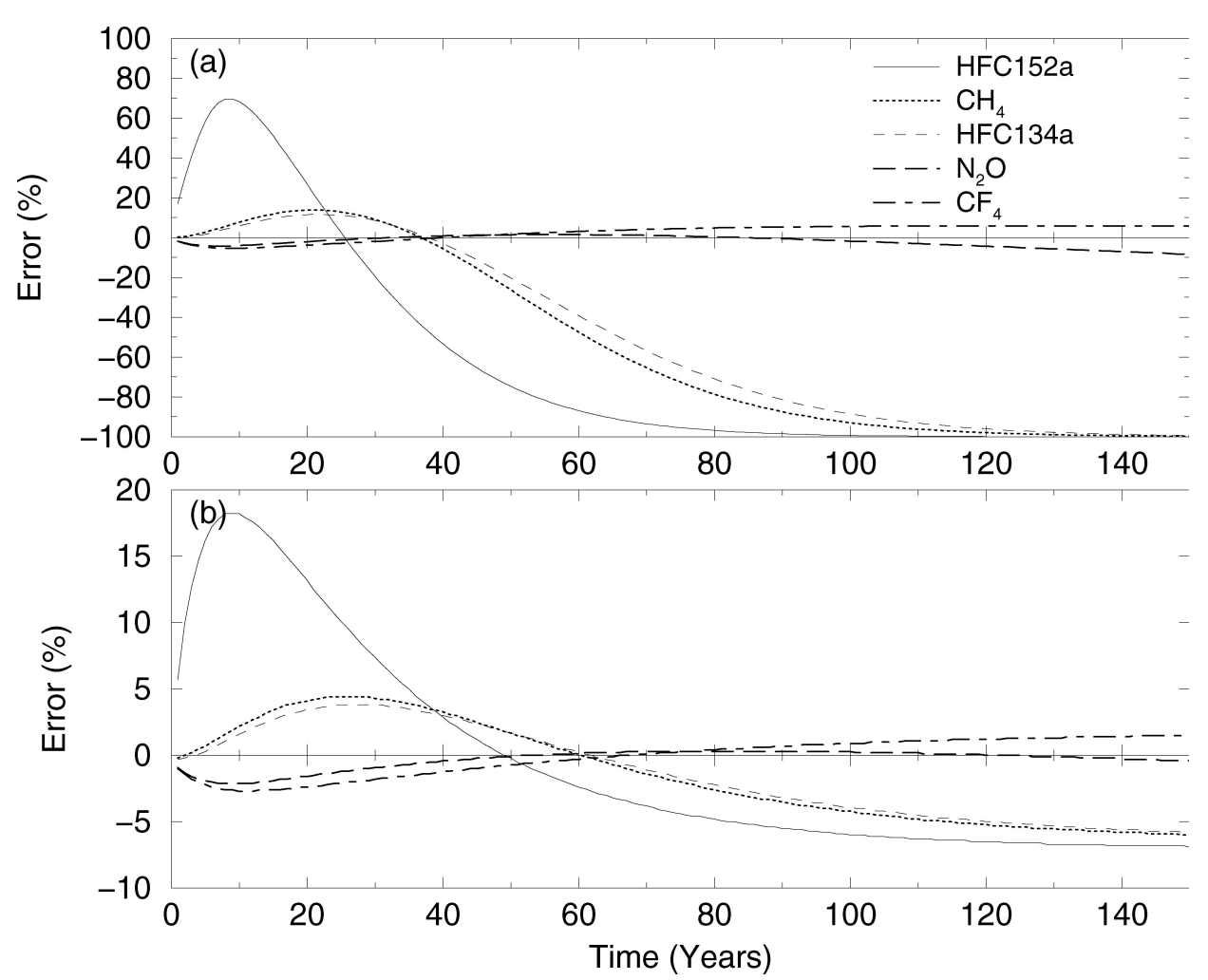

Figure 2. (a) Percentage error in the analytical $\mathrm{GTP}_{\mathrm{P}}$ relative to the $\mathrm{GTP}_{\mathrm{P}}$ calculated using the energy balance model. (b) As (a) but for the $\mathrm{GTP}_{\mathrm{S}}$.

the relative temperature impact of the pulse emission of short-lived gases for times long (compared to the gas lifetime) after the emission. Thus comparing the GWP with the EBM GTP , pulses of HFC152a, $\mathrm{CH}_{4}$ and HFC134a have a much smaller effect on temperature after 100 years than might be anticipated from the GWP; the effect reaches an order of magnitude for 500 years, as the EBM has almost "forgotten" about the pulses while the GWP still includes the integrated radiative forcing from early parts of the integration period. For $\mathrm{N}_{2} \mathrm{O}$, its relative importance at 20 and 100 years is almost identical for the GWP and GTP , although after 500 years its decay has left it with an impact only one fifth of that indicated by the GWP. For the very long-lived $\mathrm{CF}_{4}$ the two measures are qualitatively quite similar for all three time horizons.

One possibility for improving the analytical $\mathrm{GTP}_{\mathrm{P}}$ may be to represent the temperature response in Equation (2) by a sum of exponentials with different time constants representing different components of the climate system (as used, for example, by Hasselmann et al., 1997). Exploratory calculations were performed with a two-component model developed for a UNFCCC assessment (www.cru.uea.ac.uk/unfccc_assessment), which was based on the transient 
response of a general-circulation model (GCM) to a steadily increasing forcing. Such an approach yielded a value in better agreement with the EBM GTP $\mathrm{P}_{\mathrm{P}}$ at 100 years for HFC152a, although at the expense of the value at 20 years. Nevertheless, this indicates that it may be possible to achieve a better representation by fitting exponentials to a GCM response to a pulse-like forcing.

We conclude that while the simpler form of the GTP presented here may have some educational value, its performance relative to the values generated by the EBM is not adequate enough across a range of gases and time horizons for it to be considered suitable as a metric for policy purposes. However, in this paper we will continue to include results using the EBM GTP $\mathrm{P}_{\mathrm{P}}$, as the concept of temperature response to a pulse emissions nevertheless may be relevant if "exact" (see Section 1) rather than analytical values of $\mathrm{GTP}_{\mathrm{P}}$ were to be used.

\section{Performance of the GTP $_{S}$}

\subsection{ASSESSMENT RELATIVE TO THE ENERGY BALANCE MODEL}

Figure 3 shows the radiative forcing and temperature response (i.e. the $\mathrm{AGTP}_{\mathrm{S}}$ ), from the analytical model (and, for two gases, the EBM), due to the sustained emission changes. Since the concentration (and hence the forcing) comes to an equilibrium in response to the sustained emissions for times long compared to the gas lifetime, the forcing and temperature response are proportional to each other (via $\Delta T=\lambda \Delta F)$. Hence the two plots have very similar forms except at early times.

In contrast to the pulse case in Figure 1, the simple model represented by Equation (1) and the EBM show a very similar form; there is a relatively constant offset between the EBM and analytical models (Figure 3b) for both the target gas and the reference gas, so that the relative GTP $_{S}$ is quite accurate. Table II and Figure $2 b$ show the agreement for the $\mathrm{GTP}_{\mathrm{S}}$, the analytical expression and the energy balance model, for the chosen value of the heat capacity $C$. Figure $2 \mathrm{~b}$ shows agreement to better than $10 \%$ except for short time horizons for the short-lived HFC-152a; even these errors are small compared to those of the AGTP $_{P}$ shown in Figure 2a. This is encouraging for the analytical model and indicates that the GTP is likely to be a reasonably robust tool as a metric despite the simplifications in its formulation. The agreement is because the simple model is better able to represent the slow growth to the new equilibrium than it is the transient response to a pulse.

\subsection{SIMILARITY BETWEEN THE GWP AND GTP $S$}

Table II also shows that the GTP is quite similar to the GWP for all five gases in this study, a similarity that increases with time horizon. At 100 years, the time horizon chosen in the Kyoto Protocol, the GWP and analytical GTP agree to within $10 \%$. At 500 years, the agreement is around $2 \%$. Even at 20 years, the agreement is better than about $10 \%$, except for the short-lived HFC152a. 


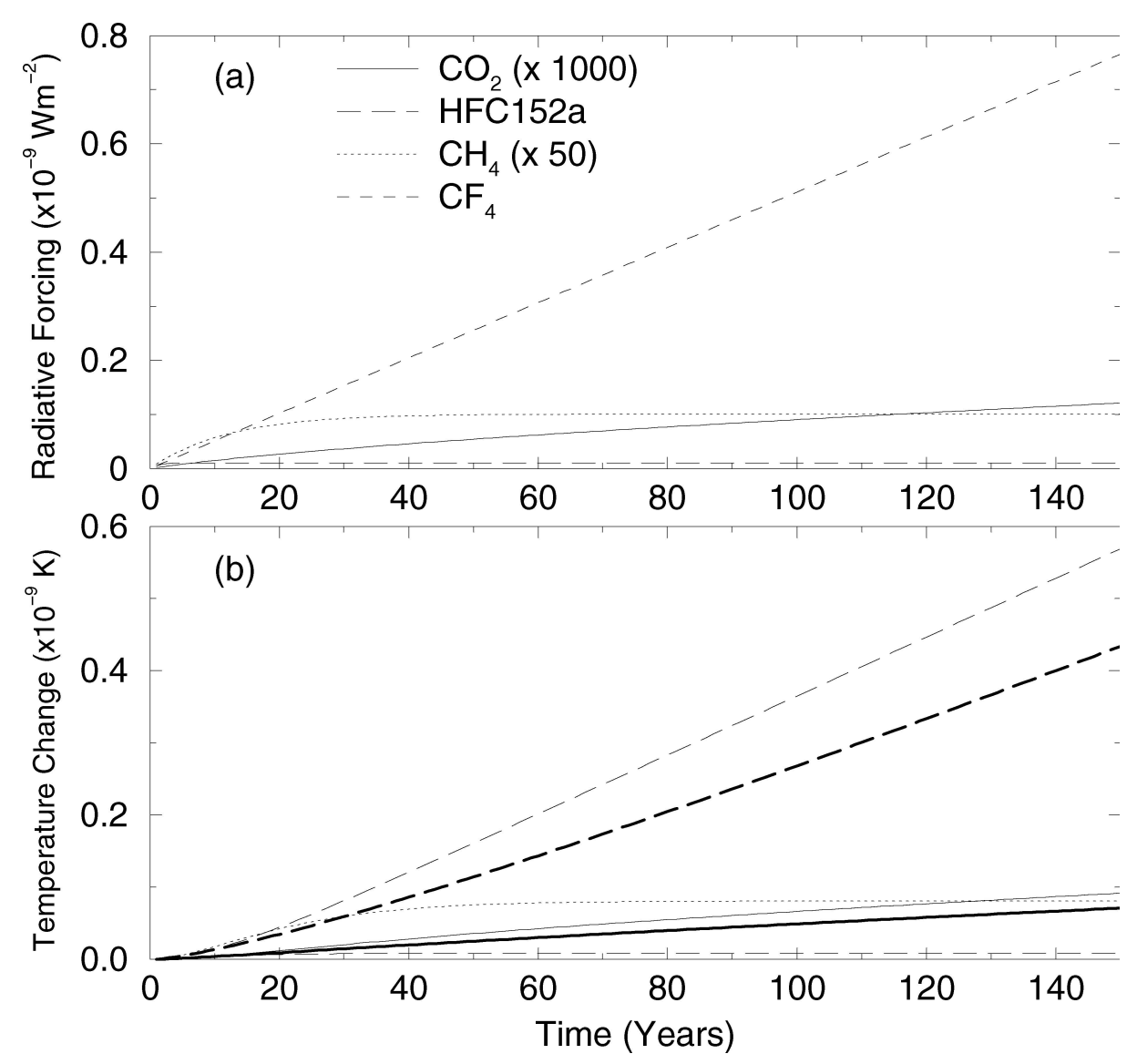

Figure 3. (a) Radiative forcing due to a sustained emission increase of $1 \mathrm{~kg} \mathrm{year}^{-1}$ of greenhouse gases with a range of lifetimes (see Table AI). (b) Temperature response using the analytical AGTP $_{S}$ to the forcings shown in (a) (thin lines). For $\mathrm{CO}_{2}$ and $\mathrm{CF}_{4}$ the response of the Energy Balance Model is also shown (thick lines).

Given that the GWP and GTP $_{S}$ are quite different conceptually, the similarity in values appears to be essentially coincidental. It can be explained by considering the asymptotic limits of the various expressions. At long time horizons, Equation (9) takes the form $A_{\mathrm{x}} \alpha_{\mathrm{x}} \lambda\left(1-\exp \left(-t / \alpha_{\mathrm{x}}\right)\right)$ which reduces to $A_{\mathrm{x}} \alpha_{\mathrm{x}} \lambda$ if the time horizon is much greater than the gas lifetime. Hence it has an identical form to Equation (A1). Similarly, Equation (11) takes the form $A_{\mathrm{c}} \lambda\left(a_{\mathrm{o}} t+\sum_{i} \alpha_{i} a_{i}\right)$ similar to the long lifetime limit of Equation (A2). Essentially, the AGWP gives the integral of a decaying pulse, while the $\mathrm{AGTP}_{\mathrm{S}}$ represents the exponential approach to an asymptotic temperature change; these two cases have the same mathematical form $\left(1-\exp \left(-t / \alpha_{\mathrm{x}}\right)\right)$ that, when ratioed with the absolute AGWP and $\mathrm{AGTP}_{\mathrm{S}}$ for $\mathrm{CO}_{2}$, yield similar values for the GWP and GTP .

This similarity between the ratio of the integrated radiative forcing of a pulse emission of two gases and the corresponding ratio of the temperature change due 
TABLE II

AGWP (in $10^{-14} \mathrm{~W} \mathrm{~m}^{-2} \mathrm{~kg}^{-1}$ year) and $\mathrm{AGTP}_{\mathrm{S}}$ (in $10^{-14} \mathrm{~K}\left(\mathrm{~kg} \text { year }{ }^{-1}\right)^{-1}$ ) from both the analytical equations and the energy balance model (EBM) for carbon dioxide and GWP and $\mathrm{GTP}_{\mathrm{S}}$ for five other greenhouse gases at time horizons of 20, 100 and $500 \mathrm{yr}$

\begin{tabular}{|c|c|c|c|c|c|c|c|c|c|}
\hline & \multicolumn{3}{|c|}{ GWP } & \multicolumn{3}{|c|}{ Analytical $\mathrm{GTP}_{\mathrm{S}}$} & \multicolumn{3}{|c|}{ EBM GTP $_{S}$} \\
\hline & 20 & 100 & 500 & 20 & 100 & 500 & 20 & 100 & 500 \\
\hline Absolute $\mathrm{CO}_{2}$ & 2.66 & 9.05 & 29.1 & 1.24 & 6.67 & 23.0 & 0.95 & 4.94 & 20.0 \\
\hline $\mathrm{HFC} 152 \mathrm{a}$ & 400 & 120 & 37 & 570 & 130 & 40 & 500 & 140 & 40 \\
\hline $\mathrm{CH}_{4}$ & 62 & 22 & 7 & 69 & 24 & 7 & 66 & 25 & 8 \\
\hline HFC134a & 3290 & 1260 & 390 & 3590 & 1370 & 400 & 3470 & 1420 & 430 \\
\hline $\mathrm{N}_{2} \mathrm{O}$ & 270 & 290 & 150 & 260 & 290 & 160 & 270 & 290 & 160 \\
\hline $\mathrm{CF}_{4}$ & 3850 & 5650 & 8730 & 3610 & 5480 & 8690 & 3700 & 5430 & 8520 \\
\hline
\end{tabular}

Note. The values for methane include the indirect forcing. The analytical GTP values are calculated with a climate sensitivity of $0.8 \mathrm{~K}\left(\mathrm{~W} \mathrm{~m}^{-2}\right)^{-1}$ and a mixed layer with a depth of $100 \mathrm{~m}$. The EBM GTP values are derived using the same climate sensitivity, with other parameters given in Appendix B.

to a sustained emission change on temperature was discussed, in terms of a steady state temperature change and infinite time horizon, in one of the earliest usages of a GWP-like metric (Fisher et al., 1990). This equivalence has been noted occasionally since then (e.g. O'Neill, 2000; Fuglestvedt et al., 2003) but has received surprisingly little attention, for example, by IPCC.

It might be argued that this similarity indicates that there is little advantage in considering replacing the GWP with the GTP . However, the fact that GTP explicitly represents the impact of a change in emissions on temperature, rather than the more abstract time integrated radiative forcing, is a substantial advantage in presenting the physical relevance of the GTP ; further, at short time horizons,

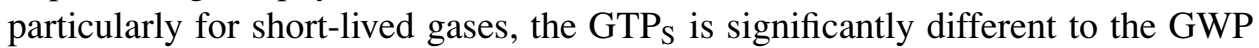
and possesses a clearer physical meaning in terms of its impact on the climate.

\section{Illustrations of the Performance of the GTP}

\subsection{COMPARISON OF THE GTP FOR GASES WITH AN IDENTICAL GWP}

One potential problem with the GWP is that two gases could have identical values for GWP(100), but different values of $A_{\mathrm{x}}$ and $\alpha_{\mathrm{x}}$, and so will have a quite different impact on the temporal evolution of the temperature change in response to a pulse emission. This is illustrated here by considering two fictitious gases, with the same GWP(100) as methane. The fictitious gases are labelled " $\mathrm{CH}_{4}$ short", with a lifetime of 4 years and a specific direct radiative forcing of $1.11 \times 10^{-3} \mathrm{~W} \mathrm{~m}^{-2} \mathrm{ppbv}^{-1}$, and " $\mathrm{CH}_{4}$ long", with corresponding values of 20 years and $2.23 \times 10^{-4} \mathrm{~W} \mathrm{~m}^{-2} \mathrm{ppbv}^{1}$. These can be compared with the IPCC-recommended values for methane of 12 years 


\section{TABLE III}

Comparison of GWP, EBM GTP , analytical GTP $_{\mathrm{S}}$ and EBM GTP at three time horizons for methane and two hypothetical methane-like gases with an identical 100-year GWP but different radiative forcings and lifetimes

\begin{tabular}{|c|c|c|c|c|c|c|c|c|c|c|c|c|}
\hline & \multicolumn{3}{|c|}{ GWP } & \multicolumn{3}{|c|}{$\mathrm{EBM} \mathrm{GTP}_{\mathrm{P}}$} & \multicolumn{3}{|c|}{ Analytical GTP } & \multicolumn{3}{|c|}{$\mathrm{EBM} \mathrm{GTP}_{\mathrm{S}}$} \\
\hline & 20 & 100 & 500 & 20 & 100 & 500 & 20 & 100 & 500 & 20 & 100 & 500 \\
\hline $\mathrm{CH}_{4}$ & 62 & 22 & 7 & 46 & 5 & 0.8 & 69 & 24 & 7 & 66 & 25 & 8 \\
\hline $\mathrm{CH}_{4}$-short & 76 & 22 & 7 & 32 & 4 & 0.8 & 99 & 24 & 7 & 90 & 26 & 8 \\
\hline $\mathrm{CH}_{4}$-long & 48 & 22 & 7 & 42 & 7 & 0.8 & 51 & 24 & 7 & 50 & 25 & 8 \\
\hline
\end{tabular}

Note. See text for details.

and $3.7 \times 10^{-4} \mathrm{~W} \mathrm{~m}^{-2} \mathrm{ppbv}^{-1}$. Since the IPCC (2001) GWP values were obtained by multiplying the specific forcing by 1.3 to account for indirect effects, the same factor is applied to the two fictitious gases to ensure that the $\operatorname{GWP}(100)=22$ for all three gases.

Table III illustrates the results. The temperature change in response to a pulse emission at $t=0$ after 100 years, as represented by the EBM GTP $\mathrm{P}_{\mathrm{P}}$, differs by about $20 \%$ between the three "gases" and they have a considerably smaller effect than might be indicated by the GWP. As expected a gas with a lower specific radiative forcing but a longer lifetime has a greater effect than a gas with a larger specific forcing and short lifetime.

However, as can be anticipated from the results in Section 4, the difference between the three gases is very small for the GTP $_{S}$ at 100 years, illustrating again the robustness of the GWP if it is reinterpreted as an approximate measure of temperature response at a given time resulting from sustained emissions. Table III also shows that the analytical and EBM generated values for GTP agree to within better than $10 \%$. The near equivalence of the GWP and GTP at $_{100}$ years does not guarantee equivalence at other time horizons, which shows that the analytical model works well in this test.

\subsection{IMPACT OF VARYING THE CLIMATE SENSITIVITY PARAMETER}

The climate sensitivity parameter $\lambda$ is one of the most uncertain features of the climate system (IPCC, 2001). It is included implicitly in the expressions for GTP via the time constant $\tau$ in Equations (9) and (11) and can be varied to illustrate the impact of the uncertainty on the potentials. We varied $\lambda$ across the IPCC (2001) range from 0.4 to $1.2 \mathrm{~K}\left(\mathrm{~W} \mathrm{~m}^{-2}\right)^{-1}$ (corresponding to double- $\mathrm{CO}_{2}$ equilibrium surface warmings of about 1.5 and $4.5 \mathrm{~K}$ respectively). Table IV illustrates

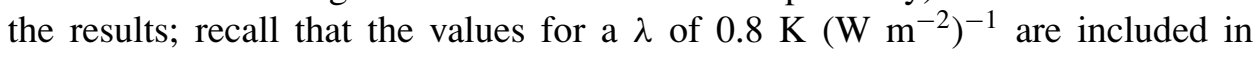
Table II.

An increased $\lambda$ yields an increased time constant $\tau$ for the climate system. However, the GTP is strikingly independent of $\lambda$, because the numerator and 


\section{TABLE IV}

Comparison of GWP, analytical GTPS and EBM GTPS at a 100 -year time horizon for two different values of the climate sensitivity parameter of 0.4 and $1.2 \mathrm{~K}\left(\mathrm{~W} \mathrm{~m}^{-2}\right)^{-1}$

\begin{tabular}{lcrrrrr}
\hline & & \multicolumn{2}{c}{ Analytical GTP } & $(100)$ & & \multicolumn{2}{c}{$\operatorname{EBM~GTP}_{\mathrm{S}}(100)$} \\
\cline { 3 - 4 } \cline { 6 - 7 } & GWP(100) & $\lambda=0.4$ & $\lambda=1.2$ & & $\lambda=0.4$ & $\lambda=1.2$ \\
\hline HFC152a & 120 & 120 & 135 & & 130 & 142 \\
$\mathrm{CH}_{4}$ & 22 & 23 & 25 & & 24 & 26 \\
$\mathrm{HFC134a}$ & 1260 & 1310 & 1420 & & 1360 & 1470 \\
$\mathrm{~N}_{2} \mathrm{O}$ & 290 & 290 & 290 & & 293 & 293 \\
$\mathrm{CF}_{4}$ & 5650 & 5560 & 5400 & & 5510 & 5380 \\
\hline
\end{tabular}

Note. Values for $\lambda=0.8 \mathrm{~K}\left(\mathrm{~W} \mathrm{~m}^{-2}\right)^{-1}$ are given in Table II.

denominator are, to first order, affected in a similar way by changes in $\lambda$. Table IV shows that the GTP $_{S}$ varies by only about $10 \%$ between the extremes of $\lambda$ for the short-lived HFC152a, with the variation decreasing markedly for the longer lived gases. Table IV also shows that the analytical $\mathrm{GTP}_{\mathrm{S}}$ and the EBM GTP $\mathrm{S}_{\mathrm{S}}$ also agree equally well at all the tested values of $\lambda$. Further tests showed that this good agreement was also found at time horizons of 20 and 500 years.

Hence, the uncertainty in $\lambda$ is not a barrier to developing a relative metric based on temperature change.

\subsection{VERY SHORT-LIVED SPECIES}

The GWP has not generally been applied to the climate effect of very shortlived species (order of weeks or less), such as those of very short-lived greenhouse gases, sulphur dioxide, tropospheric ozone precursors or aircraft-induced contrails. Reasons for this omission appear to include the fact that emissions of short-lived gases yield much more inhomogeneous changes in concentration and the fact that they often cause significant, albeit highly uncertain, indirect effects on other radiatively important constituents. In principal, accounting for these two facts is not an insuperable problem. The absence of values of metrics for these species could form a barrier to their inclusion in future protocols of the UNFCCC. The $\mathrm{GTP}_{\mathrm{S}}$ formulation does allow a framework to consider their inclusion. Note that the $\operatorname{GTP}_{\mathrm{P}}$ for such gases would be very small for any time horizon beyond a year or so and thus is not considered here.

For a short-lived species, the forcing is almost immediately in equilibrium with the emission change so that Equation (9) becomes

$$
\operatorname{AGTP}_{\mathrm{S}}^{\text {short }}(t)=\lambda \alpha_{\mathrm{X}} A_{\mathrm{x}}\left[1-\exp \left(-\frac{t}{\tau}\right)\right] .
$$


As an example, we take $\alpha_{\mathrm{x}} A_{\mathrm{x}}$ due to the direct effect of sulphate aerosol to be $-5 \times 10^{-12} \mathrm{~W} \mathrm{~m}^{-2}\left(\mathrm{kgS} \mathrm{yr}^{-1}\right)^{-1}$ (derived from values in IPCC (2001), assuming $80 \mathrm{TgS} \mathrm{yr}^{-1}$ of anthropogenic emissions cause a forcing of $-0.4 \mathrm{~W} \mathrm{~m}^{-2}$, and that the forcing is linear in emissions). This then yields GTP $_{S}$ at 20,100 and 500 years of $-240,-52$ and -15 respectively, indicating the extent to which the (direct) cooling due to a constant emission of sulphur is more effective in changing global-mean temperature than the same emission of $\mathrm{CO}_{2}$.

Of course, there are considerable hurdles to a rigorous calculation of $\mathrm{GTP}_{\mathrm{S}}$ for sulphate, as its full impact depends on the presence of other aerosol components, on the highly uncertain impact of sulphate on cloud radiative properties and on the time and location of the emissions (IPCC, 2001). The same is true for emissions of, for example, $\mathrm{NO}_{\mathrm{x}}$. Nevertheless, in a policy context, it may well be decided that an approximate index is better than no index at all, and the GTP $\mathrm{S}_{\mathrm{S}}$ provides a possible framework for consideration. And, as importantly, many of the difficulties present in quantifying these forcings are also present when EBMs are used to generate temperatures for future scenarios (for example in IPCC, 2001) and in GCMs. Hence, the difficulty in quantifying the forcings for these short-lived species is not a problem confined to the calculation of the GTPS.

\section{Conclusions}

In the preparations for both the next IPCC assessment and the negotiations for the second commitment period of the Kyoto Protocol, it is important that the climate change community reassesses the metrics available to policymakers for comparing the climatic impacts of different emissions.

In this paper, as a contribution to this reassessment, two related alternatives to the GWP have been presented, which represent the impacts of emissions on globalmean surface temperature change. The $\mathrm{GTP}_{\mathrm{P}}$ compares the temperature effect of pulse emissions, while the $\mathrm{GTP}_{\mathrm{S}}$ compares the effect of sustained emission changes. Both new metrics retain some of the attractions of the GWP, such as a transparent formulation, the reliance on relatively few parameters and the possibility of use by policymakers with little further input from scientists. They have a clear advantage over the GWP in that they represent an actual (if crude) climate impact, rather than the more abstract concept of integrated radiative forcing due to a pulse emission.

The simple analytical expressions derived for the $\mathrm{GTP}_{\mathrm{P}}$ are shown to perform poorly compared to an energy balance model and are not considered to be appropriate for consideration for policy purposes. However, despite the failure of the analytical form of the $\mathrm{GTP}_{\mathrm{P}}$, the concept remains a valid one and a useful way of comparing the temperature impact of pulse emissions.

The GTP $_{S}$ may be a more policy-relevant metric as it represents the relative climate impact of sustained emission changes. One interesting result is that the GTP $_{S}$ and GWP produce similar values, a result that was essentially pointed out in a somewhat different context by Fisher et al. (1990). This result indicates that the 
GWP may have a greater utility than has generally been recognised and represents more than just the integrated radiative forcing in response to a pulse emission. Nevertheless, the $\mathrm{GTP}_{\mathrm{S}}$ more explicitly represents the temperature response to a sustained emission change for all time horizons and would be preferable to a reinterpreted GWP, if a temperature change metric is required. The $\mathrm{GTP}_{\mathrm{S}}$ could then be used in a similar way to the GWP, as a basis for providing equivalence between sustained emission changes of $\mathrm{CO}_{2}$ and other greenhouse gases for the period up to a given time (e.g. 100 years) in the future.

Unlike the $\mathrm{GTP}_{\mathrm{P}}$, the $\mathrm{GTP}_{\mathrm{S}}$ reproduces results from an energy balance model to better than about $10 \%$ at 20,100 and 500 years and is also relatively insensitive to uncertainties in the climate sensitivity parameter; this uncertainty, to first order, affects the gas under consideration and the reference gas in a similar way. This lack of sensitivity would certainly aid the adoption of the $\mathrm{GTP}_{\mathrm{S}}$ as a replacement to the GWP.

If the $\operatorname{GTP}_{\mathrm{S}}$ was to find favour as a metric, its similarity with the GWP can be considered as an advantage, as the policy consequences of changing the metric would be slight (assuming, of course, that a time horizon of 100 years is retained for the GTP , which is by no means obvious). One aspect and possible drawback of the GTP (and the GWP when reinterpreted as an approximate GTP G $_{S}$ is that emission changes must be maintained beyond any reporting period; otherwise a GTP $_{S}$-based equivalent emission change would not guarantee (approximate) equivalence in terms of induced temperature change if the chosen time horizon is beyond the reporting period.

The GTP ${ }_{P}$ and GTP $_{S}$ formulations presented here could no doubt be extended further-for example, multiple timescales of climate response could be represented by using a sum of exponentials approach. It is possible that values could be derived for emission changes intermediate between pulse and sustained (e.g. Smith, 2003), which might be useful in some applications. The formulation could also be extended to represent other parameters, such as sea-level rise, the rate of temperature changes or even economic damage. One aspect of the GTP developed here is that it concentrates on equivalence of temperature change at a particular time, rather than the path of temperature change leading up that time. A further possible extension of the metric would be towards one that used integrated temperature change up to a given time horizon, which would account, to some extent, for the path.

One significant simplification in this study is the representation of the carbon budget. It may be that in any "legislative" application of the GTP, a more sophisticated carbon budget model could be used to provide the AGTP for the reference gas and that model could also have a built-in dependence on a chosen background scenario of $\mathrm{CO}_{2}$ change. The same is true for the other greenhouse gases. Indeed, as discussed in Section 1, it may be that "exact" values generated by a numerical model that explicitly accounts for these factors might be favoured over values generated by the analytical approach presented here; the loss of simplicity and transparency 
would have to be balanced against what would be a more faithful representation of the true climate system response.

\section{Appendix A: Calculation of the GWP and Specification of Gas-Dependent Parameters}

This appendix gives some basic information on parameters used in this work, to allow the calculation of the values here. For consistency, all values used for GWP calculations are essentially identical to those in IPCC (2001).

The absolute global warming potential for non- $\mathrm{CO}_{2}$ greenhouse gases is given by

$$
\operatorname{AGWP}^{\mathrm{x}}(t)=\int_{0}^{t} A_{\mathrm{x}} \exp \left(-t^{\prime} / \alpha_{\mathrm{x}}\right) d t^{\prime}=A_{\mathrm{x}} \alpha_{\mathrm{x}}\left[1-\exp \left(-\frac{t}{\alpha_{\mathrm{x}}}\right)\right] .
$$

For carbon dioxide, using Equation (4) then

$$
\begin{aligned}
\operatorname{AGWP}^{\mathrm{C}}(t) & =\int_{0}^{t} A_{\mathrm{c}}\left[a_{\mathrm{o}}+\sum_{i} a_{i} \exp \left(-\frac{t^{\prime}}{\alpha_{i}}\right)\right] d t^{\prime} \\
& =A_{\mathrm{c}}\left[a_{\mathrm{o}} t+\sum_{i} a_{i} \alpha_{i}\left(1-\exp \left(-\frac{t}{\alpha_{i}}\right)\right)\right] .
\end{aligned}
$$

For Equations (A2) and (4), the coefficients are $a_{\mathrm{o}}=0.1756, a_{1}=0.1375, a_{2}=$ $0.1858, a_{3}=0.2423$ and $a_{4}=0.2589 . \alpha_{1}=421.093, \alpha_{2}=70.5965, \alpha_{3}=$ $21.4216, \alpha_{4}=3.4154$ (all $\alpha$ values are in years). These coefficients were provided by F. Joos (2003, personal communication) using the model of Joos et al. (1996) and are a fit to the same response function as used by IPCC (2001). However, note that IPCC used a fit with a different mathematical form (WMO, 1999) that was not amenable to the analytical integrations performed in this paper. The difference in the two fits results in a slight difference in the values for the AGWP ; for example, at 100 years, the value used here is $1.5 \%$ higher than the IPCC (2001) value, and consequently some of the values of GWP also slightly differ from (and are slightly lower than) the IPCC (2001) values. Table AI lists the values of $A_{\mathrm{x}}$ and $\alpha_{\mathrm{x}}$ used here.

For Equations (3), (5), (9), (11), (A1) and (A2), some care is required with the units. It is convention, in IPCC reports and elsewhere, to quote the specific radiative forcings in $\mathrm{W} \mathrm{m}^{-2} \mathrm{ppbv}^{-1}$, whereas the expressions use a mass form in $\mathrm{W} \mathrm{m}{ }^{-2} \mathrm{~kg}^{-1}$; values for both forms are shown in Table A1. To convert the per ppbv values to per $\mathrm{kg}$, they must be multiplied by $\left(M_{\mathrm{A}} / M_{\mathrm{x}}\right)\left(10^{9} / T_{\mathrm{M}}\right)$ where $M_{\mathrm{A}}$ is the mean molecular weight of air $\left(28.96 \mathrm{~kg} \mathrm{kmol}^{-1}\right), M_{\mathrm{x}}$ is the molecular weight of molecule $\mathrm{x}$, and $T_{\mathrm{M}}$ is the total mass of the atmosphere, about $5.15 \times 10^{18}$ $\mathrm{kg}$. 
TABLE AI

Values of radiative forcing, $A_{\mathrm{x}}$, (in $\mathrm{W} \mathrm{m}^{-2} \mathrm{~kg}^{-1}$ and $\mathrm{W} \mathrm{m}^{-2} \mathrm{ppbv}^{-1}$ ) and gas lifetime, $\alpha_{\mathrm{x}}$, (in yr) from IPCC (2001) for the gases used in this paper

\begin{tabular}{llll}
\hline Gas & $\begin{array}{l}\text { Radiative forcing } \\
\left(\mathrm{W} \mathrm{m}^{-2} \mathrm{~kg}^{-1}\right)\end{array}$ & $\begin{array}{l}\text { Radiative forcing } \\
\left(\mathrm{W} \mathrm{m}^{-2} \mathrm{ppbv}^{-1}\right)\end{array}$ & Lifetime $(\mathrm{yr})$ \\
\hline $\mathrm{CO}_{2}$ & $1.98 \times 10^{-15}$ & $1.548 \times 10^{-5}$ & See text \\
$\mathrm{HFC152a}_{\left(\mathrm{CH}_{3} \mathrm{CHF}_{2}\right)}$ & $7.66 \times 10^{-12}$ & 0.09 & 1.4 \\
$\mathrm{CH}_{4}$ & $1.3 \times 10^{-13}$ & $3.7 \times 10^{-4}$ & 12 \\
$\mathrm{HFC134a}\left(\mathrm{CH}_{2} \mathrm{FCF}_{3}\right)$ & $8.27 \times 10^{-12}$ & 0.15 & 13.8 \\
$\mathrm{~N}_{2} \mathrm{O}$ & $3.96 \times 10^{-13}$ & $3.1 \times 10^{-3}$ & 114 \\
$\mathrm{CF}_{4}$ & $5.11 \times 10^{-12}$ & 0.08 & 50,000 \\
\hline
\end{tabular}

Note. The radiative forcing for methane given in this table has to be multiplied by 1.3 to account for indirect forcings in the calculations presented here.

In addition, if the time constants $\tau$ and $\alpha$ are taken to be in years, then it is necessary to multiply Equations (3), (5), (9) and (11) by the number of seconds in a year, about $3.16 \times 10^{7} \mathrm{~s}$.

\section{Appendix B: Energy Balance Model}

To evaluate the analytical forms of the $\mathrm{GTP}_{\mathrm{P}}$ and $\mathrm{GTP}_{\mathrm{S}}$ we employ an energy balance model which resolves hemispheres and land/ocean and represents heat exchange in the ocean using an upwelling-diffusion model similar in structure to that used with IPCC (2001) (see especially Appendix 9.1 of that report). The basic structure of such models has been described by, for example, Hoffert et al. (1980), Harvey and Schneider (1985), Wigley and Raper (1993) and Raper et al. (2001) and will not be repeated here. The parameters chosen here are representative of mid-range values used in the above literature.

The ocean mixed-layer depth is taken to be $100 \mathrm{~m}$, and the deep ocean $4 \mathrm{~km}$, with a diffusive layer of $1 \mathrm{~km}$. The vertical grid spacing in the ocean is $100 \mathrm{~m}$. The default ocean upwelling velocity is taken to be $4 \mathrm{~m} \mathrm{year}^{-1}$, but incorporates a linear dependence on the change in mixed-layer temperature to mimic the effect of weakening of the thermohaline circulation, following $\operatorname{IPCC}(1996,2001)$; a value of change of $12{ }^{\circ} \mathrm{C}$ is taken as the point at which thermohaline circulation collapse occurs and this acts to reduce the upwelling velocity by $30 \%$. The oceanic diffusion coefficient is $1.5 \mathrm{~cm}^{2} \mathrm{~s}^{-1}$ and the polar parameter is 0.2 . The depth of thermally interactive land is $2.3 \mathrm{~m}$. The ocean-land and northern-southern hemisphere exchange coefficients are taken to be $0.8 \mathrm{~W} \mathrm{~m}^{-2}\left({ }^{\circ} \mathrm{C}\right)^{-1}$, and the land/ocean climate sensitivity ratio is 1.3 . A time step of 3 days is used. The climate sensitivity parameter is an input parameter and, unless otherwise noted, has the value $0.8 \mathrm{~W} \mathrm{~m}^{-2}\left({ }^{\circ} \mathrm{C}\right)^{-1}$. 


\section{Acknowledgements}

This work was initiated as part of the European Community project METRIC (EVK2-CT-1999-00021). KPS completed it under UK Natural Environment Research Council grant NER/L/S/2001/00661, and JSF completed it under the project "Modelling impacts of climate policy" funded by the Norwegian Research Council. We are grateful to Terje Berntsen, Gylvan Meira Filho, Bård Romstad and Robert Sausen for comments and encouragement. Steven Smith is thanked for a particularly detailed and perceptive review that led to significant improvements.

\section{References}

Fisher, D. A., Hales, C. H., Wang, W. -C., Ko, M. K. W. and Sze, N. D.: 1990, 'Model calculation on the relative effects of CFCs and their replacements on global warming', Nature 344, 513-516.

Fuglestvedt, J. S., Berntsen, T. K., Godal, O., Sausen, R., Shine, K. P. and Skodvin, T.: 2003, 'Metrics of climate change: Assessing radiative forcing and emission indices', Clim Change 58, 267331.

Fuglestvedt, J. S., Berntsen, T., Godal, O. and Skodvin, T.: 2000, 'Climatic implications of GWP-based reductions in greenhouse gas emissions', Geophys. Res. Lett. 27, 409-412.

Godal, O.: 2003, 'The IPCC's assessment of multidisciplinary issues: The case of greenhouse gas indices', Clim. Change 58, 243-249.

Hammitt, J. K., Jain, A. K., Adams, J. L. and Wuebbles, D. J.: 1996, 'A welfare-based index for assessing environmental effects of greenhouse-gas emissions', Nature 381, 301-303.

Hansen, J., Sato, M. and Ruedy, R.: 1997, 'Radiative forcing and climate response', J. Geophys. Res. Atmos. 102, 6831-6864.

Hartmann, D. L.: 1996, Global Physical Climatology, Academic Press, New York.

Harvey, L. D. D. and Schneider, S. H.: 1985, 'Transient climate response to external forcing on $10^{0}-10^{4}$ year time scales, Part 2 : Sensitivity experiments with a seasonal, hemispherically averaged, coupled atmospheric, land, and ocean energy balance model, J. Geophys. Res. 90, 22072222.

Hasselmann, K., Hasselmann, S., Giering, R., Ocana, V. and van Storch, H.: 1997, 'Sensitivity study of optimal $\mathrm{CO}_{2}$ emission paths using a structural integrated assessment model (SIAM)', Clim. Change 37, 345-386.

Hoffert, M. I., Callegari, A. J. and Hseih, C. T.: 1980, 'The role of the deep sea heat storage in the secular response to climate forcing', J. Geophys. Res. 85, 6667-6679.

The Intergovernmental Panel on Climate Change (IPCC): 1990, Climate Change: The Intergovernmental Panel on Climate Change Scientific Assessment, Cambridge University Press, Cambridge, UK.

IPCC: 1995, Climate Change 1994. The Intergovernmental Panel on Climate Change, Cambridge University Press, Cambridge, UK.

IPCC: 1996, Climate Change 1995. The Science of Climate Change. The Intergovernmental Panel on Climate Change, Cambridge University Press, Cambridge, UK.

IPCC: 2001, Climate Change 2001: The Scientific Basis. Intergovernmental Panel on Climate Change, Cambridge University Press, Cambridge, UK.

Joos, F., Bruno, M., Fink, R., Stocker, T. F., Siegenthaler, U., Le Quéré, C. and Sarmiento, J. L.: 1996, 'An efficient and accurate representation of complex oceanic and biospheric models of anthropogenic carbon uptake', Tellus 48B, 397-417. 
Joshi, M. M., Shine, K. P., Ponater, M., Stuber, N., Sausen, R. and Li, L.: 2003, 'A comparison of climate response to different radiative forcings in three general circulation models: Towards an improved metric of climate change', Clim. Dyn. 20, 843-854.

Kandlikar, M.: 1995, 'The relative role of trace gas emissions in greenhouse gas abatement policies', Energy Policy 23, 879-883.

Manne, A. S. and Richels, R. G.: 2001, 'An alternative approach to establishing trade-offs among greenhouse gases', Nature 410, 675-677.

Meira Filho, L. G. M. and Miguez, J. D. G.: 2000, 'Note on the time-dependent relationship between emissions of greenhouse gases and climate change', Technical Note of the Ministry of Science and Technology, Federative Republic of Brazil, Available at: www.mct.gov.br/cli $\mathrm{ma} /$ ingles/negoc/proposta.htm, July 2004.

O'Neill, B. C.: 2000, 'The jury is still out on global warming potentials', Clim. Change 44, 427443.

O'Neill, B. C.: 2003, 'Economics, natural science, and the costs of the global warming potential', Clim. Change 58, 251-260.

Raper, S. C. B., Gregory, J. M. and Osborn, T. J.: 2001, 'Use of an upwelling diffusion energy balance model to simulate and diagnose A/OGCM results', Clim. Dyn. 17, 601-613.

Sausen, R. and Schumann, U.: 2000, 'Estimates of the climate response to aircraft $\mathrm{CO}_{2}$ and $\mathrm{NO}_{\mathrm{x}}$ emission scenarios', Clim. Change 44, 27-58.

Schmalensee, R.: 1993, 'Comparing greenhouse gases for policy purposes', Energy J. 14, 245-255.

Sihra, K., Hurley, M. D., Shine, K. P. and Wallington, T. J.: 2001, 'Updated radiative forcing estimates of sixty-five halocarbons and non-methane hydrocarbons', J. Geophys. Res. 106, 2049320505.

Skodvin, T. and Fuglestvedt, J. S.: 1997, 'A comprehensive approach to climate change: Political and scientific considerations', Ambio 26, 351-358.

Smith, S. J.: 2003, 'The evaluation of greenhouse gas indices', Clim. Change 58, 261-265.

Smith, S. J. and Wigley, T. M. L.: 2000a, 'Global warming potentials, 1: Climatic implications of emissions reductions', Clim. Change 44, 445-457.

Smith, S. J. and Wigley, T. M. L.: 2000b, 'Global warming potentials, 2: Accuracy', Clim. Change 44, 459-469.

Wigley, T. M. L.: 1998, 'The Kyoto protocol: $\mathrm{CO}_{2}, \mathrm{CH}_{4}$ and climate implications', Geophys. Res. Lett. 25, 2285-2288.

Wigley, T. M. L. and Raper, S. C. B.: 1993, 'Future changes in global-mean temperature and sea level. In: Warrick, R. A., Barrow, E. M. and Wigley, T. M. L. (eds.), Climate and Sea Level Change: Observations, Projections and Implications, Cambridge University Press, Cambridge, UK.

WMO: 1999, Scientific Assessment of Ozone Depletion: 1998: Global Ozone Research and Monitoring Project Report No 44, World Meteorological Organization, Geneva, Switzerland.

Wuebbles, D. J., Jain, A. K., Patten, K. O. and Grant, K. E.: 1995, 'Sensitivity of direct global warming potentials to key uncertainties', Clim. Change 29, 265-297.

(Received 28 April 2003; in revised form 19 May 2004) 